\title{
Enzyme Active Site Loop Revealed as a Gatekeeper for Cofactor Flip by Targeted Molecular Dynamics Simulations and FRET-Based Kinetics
}

\author{
Sophie Rahuel-Clermont, ${ }^{* \dagger} \dagger$ Raphaël Bchini, ${ }^{\dagger}{ }^{\S}$ Sophie Barbe, ${ }^{\ddagger}$ Séverine Boutserin, ${ }^{\dagger}$ Isabelle André, ${ }^{\ddagger}$ (i) \\ and François Talfournier*, ${ }^{\dagger}$ \\ ${ }^{\dagger}$ Université de Lorraine, CNRS, IMoPA, Campus Biologie Santé, F-54000 Nancy, France \\ ${ }^{\ddagger}$ Laboratoire d’Ingénierie des Systèmes Biologiques et Procédés, LISBP, Université de Toulouse, CNRS, INRA, INSA, Toulouse, \\ France, 135, Avenue de Rangueil, F-31077 Toulouse cedex 04, France
}

Supporting Information

ABSTRACT: Structural motions are key events in enzyme catalysis, as exemplified by the conformational dynamics associated with the cofactor in the catalytic mechanism of hydrolytic $\mathrm{NAD}(\mathrm{P})$-dependent aldehyde dehydrogenases. We previously showed that, after the oxidoreduction step, the reduced cofactor must adopt a flipped conformation, which positions the nicotinamide in a conserved cavity that might constitute the exit door for $\operatorname{NAD}(\mathrm{P}) \mathrm{H}$. However, the molecular basis that make this movement possible is unknown. Based on the pre- and postflip Xray structures, targeted molecular dynamic simulations enabled us to identify the $\mathrm{E}^{268} \mathrm{LGG}^{271}$ conserved loop that must shift to allow reduced nicotinamide conformational switch. To monitor cofactor
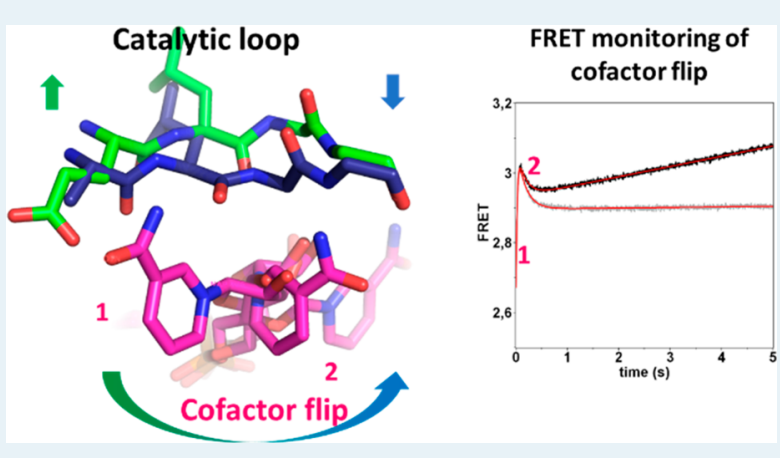
movements within the active site, we used an intrinsic fluorescence resonance energy transfer signal between Trp177 and the reduced nicotinamide moiety to kinetically track the flip during the catalytic cycle of retinal dehydrogenase 2 (ALDH1A2). Decreasing loop flexibility by substituting Ala for Gly271 drastically reduced the rate constant associated with this movement that became rate-limiting. We thus propose that the $\mathrm{E}^{268} \mathrm{LGG}^{271}$ loop acts as a gatekeeper for cofactor flipping. Similar approaches applied to a CoA-dependent aldehyde dehydrogenase showed that cofactor flipping likely extends to the whole ALDH family, thus bridging the gap between the well-studied chemical steps and a conformational transition essential for catalysis.

KEYWORDS: enzyme catalysis, cofactor flip, targeted molecular dynamic simulations, kinetics, FRET, aldehyde dehydrogenase

\section{INTRODUCTION}

Structural flexibility and conformational rearrangements possibly coupled with substrate and cofactor are recognized as key players in enzyme mechanisms (see, e.g., refs $1-6$ ). This notion not only is essential for our fundamental understanding of enzyme catalysis but also for tailoring biotechnologically relevant enzymes. ${ }^{7}$ However, designing experimental approaches for such studies remains challenging, ${ }^{2,8-10}$ especially when small local motions are at play and requires integrated approaches that combine kinetic, structural, and molecular dynamics studies.

The aldehyde dehydrogenases (ALDHs) exemplify such mechanisms as multiple convergent reports correlate local motions to several catalytic steps and are thus very good models to address these central issues. They catalyze the $\mathrm{NAD}(\mathrm{P})$-dependent oxidation of a wide variety of aldehydes to their corresponding nonactivated or CoA-activated acids and participate in many biological processes, such as intermediary metabolism, detoxification, osmotic protection, and cellular differentiation. ${ }^{11}$ In humans, ALDHs are linked to severe diseases including cancers and developmental defects. ${ }^{11-15}$ They share a similar two-step chemical mechanism comprising an acylation and a deacylation that depends on the nature of the acyl acceptor (Figure 1A). Compared to their hydrolytic counterparts that follow an ordered sequential mechanism in which $\mathrm{NAD}(\mathrm{P}) \mathrm{H}$ dissociates last, ${ }^{16,17}$ the CoA-dependent ALDHs obey a ping pong mechanism, which involves early $\mathrm{NADH}$ release prior to deacylation by CoA (i.e., transthioesterification) ${ }^{18-20}$ In addition, the reaction catalyzed by methylmalonate semialdehyde dehydrogenase (MSDH) includes a $\beta$-decarboxylation process which arises after $\mathrm{NADH}$ release (Figure 1A).

In ALDHs, local dynamics are associated with formation of an enzyme-substrate-cofactor ternary complex competent for acylation. This is the case, e.g., for nonphosphorylating glyceraldehyde 3-phosphate dehydrogenase (GAPN) that 

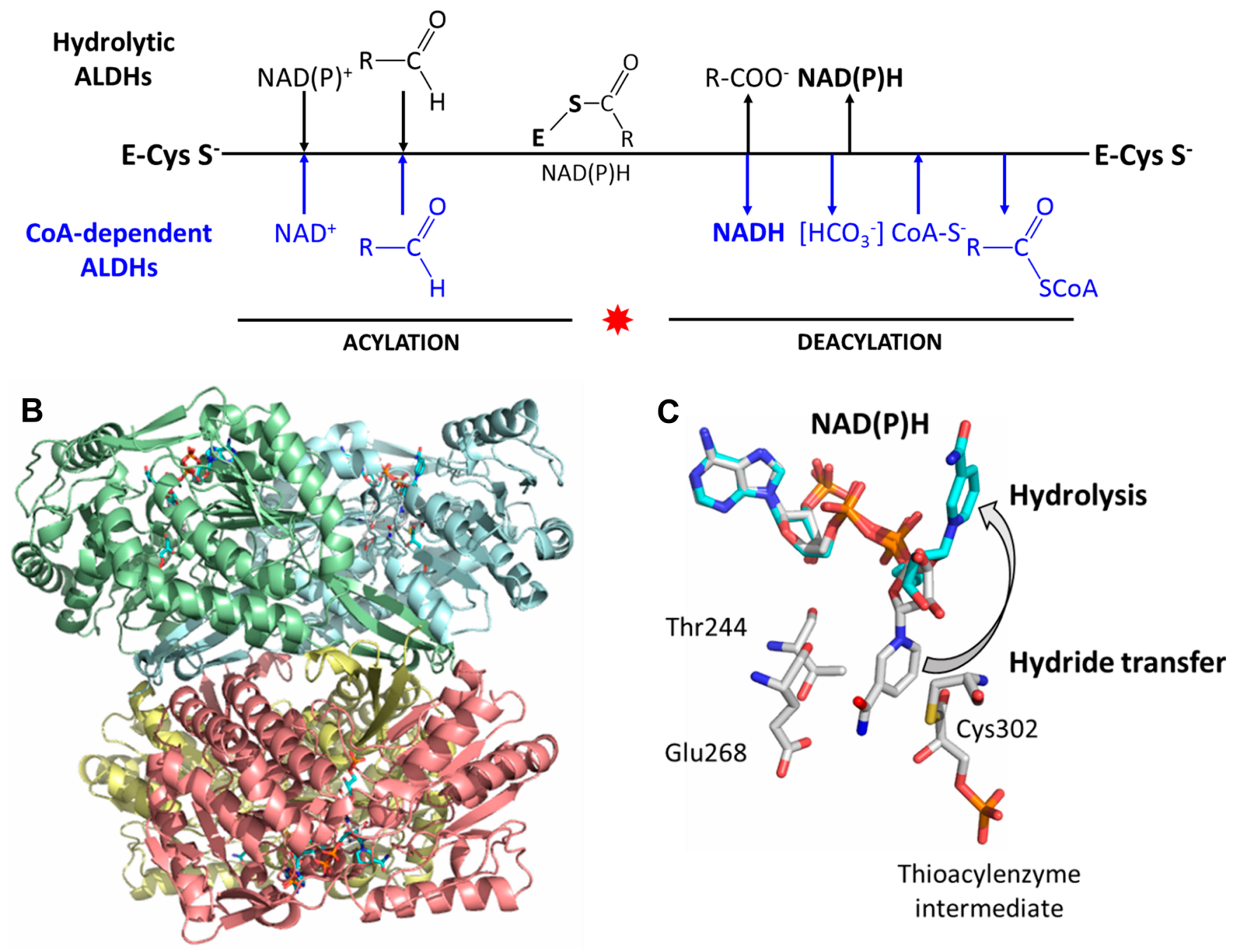

Thioacylenzyme

Figure 1. Mechanistic and structural description of cofactor NMNH flip in ALDHs. (A) Cleland-type scheme of hydrolytic and CoA-dependent ALDHs kinetic mechanism identifying the flip event (red star). The acylation step involves nucleophilic attack of the catalytic Cys302 (E-CysS ${ }^{-}$) on the aldehyde function followed by hydride transfer leading to the formation of a thioacylenzyme intermediate and NAD $(\mathrm{P}) \mathrm{H}$. The deacylation step includes the attack of a water or CoA molecule on the nascent thioacylenzyme intermediate and release of the nonactivated or CoA-activated acids. The reaction catalyzed by $\mathrm{MSDH}$ includes additional $\beta$-decarboxylation ( $\mathrm{HCO}_{3}{ }^{-}$release). (B) GAPN tetramer, showing location the active sites, and (C) active site zoom structures showing NADPH in hydride transfer conformation, Thr244 and Glu268 (PDB code 2euh), superimposed with the thioacylenzyme intermediate and NADPH in deacylation conformation (PDB code 2qe0).

undergoes ligand-induced thermal destabilization ${ }^{21}$ and retinal dehydrogenase 2 (ALDH1A2) whose substrate specificity is ensured by a disorder to order transition..$^{22,23}$ In hydrolytic ALDHs, cofactor binding mode and motions control deacylation to some extent. ${ }^{24-28}$ Indeed, the presence of multiple conformations of the nicotinamide mononucleotide (NMN) moiety is supported by the majority of the X-ray structures of ALDH-NAD(P) binary complexes ${ }^{29-31}$ as well as NMR studies. ${ }^{32}$ During the acylation, the $\beta$-methyl group of the invariant conserved $\mathrm{Thr} 244$ residue allows the nicotinamide ring to adopt a productive conformation for hydride transfer $^{33}$ (Figure 1B,C). Nevertheless, this conformation is not suitable for the deacylation because it would sterically preclude the catalytic Glu268 from activating the water molecule in the hydrolytic process. Because the cofactor remains bound to the enzyme along the reaction coordinate, a movement of the reduced NMN (NMNH) moiety of the $\mathrm{NAD}(\mathrm{P}) \mathrm{H}$ is thus a prerequisite for completion of the second half of the reaction. ${ }^{27}$ The crystal structure of a GAPN thioacylenzyme intermediate/NADPH complex provided an essential clue in revealing a flip of the NMNH moiety, which positions the reduced nicotinamide in a conserved $\mathrm{NAD}(\mathrm{P}) \mathrm{H}$ exit pocket, without significant changes of the protein structure. ${ }^{34,35}$ Therefore, although the initial and final conformations of the cofactor are defined, available structural data did not reveal how this movement occurred nor the driving force that made it possible.

To decipher the precise conformational transition of $\mathrm{NMNH}$ at atomic level and to identify potential role of coupled protein dynamics in driving this movement, we associated targeted molecular dynamics (TMD) to simulate the conformational transition of the cofactor and real-time monitoring of the NMNH conformational changes within the active site using an intrinsic fluorescence resonance energy transfer (FRET) probe. We identified a short active site loop 
that acts as a gatekeeper for cofactor flipping, a feature that likely extends to the whole ALDH superfamily.

\section{EXPERIMENTAL PROCEDURES}

Materials. $\mathrm{NAD}^{+}$and $\mathrm{NADH}$ were purchased from Roche (Mannheim, Germany). Decanal, Hepes, Tween 80, pyruvic acid and lactate dehydrogenase from rabbit muscle (LDH) were sourced from Sigma-Aldrich (St. Louis, MO, U.S.A.) and Coenzyme A (CoA) from Calbiochem (Merck, Darmstadt, Germany). Stock solution of decanal $(100 \mu \mathrm{M})$ was prepared in acetonitrile.

Site-Directed Mutagenesis, Production, and Purification of Wild-Type and Mutated ALDHs. Mutated enzymes were generated by standard PCR site-directed mutagenesis. Recombinant Rattus norvegicus testis ALDH1A2 was produced as $\mathrm{N}$-terminal His-tagged fusion, hereafter referred to as ALDH1A2, as described previously. ${ }^{23}$ Briefly, Escherichia coli $\mathrm{C} 41$ (DE3) transformants were grown at $37{ }^{\circ} \mathrm{C}$ for $16 \mathrm{~h}$ in the ZYM5052 or N5052 autoinducible medium ${ }^{36}$ supplemented with ampicillin $(200 \mathrm{mg} / \mathrm{L})$ and purified by high performance nickel-Sepharose chromatography using a $0.5 \mathrm{M}$ imidazole elution step. Enzyme concentration was determined spectrophotometrically using a molar absorption coefficient of 2.11 $\times 10^{5} \mathrm{M}^{-1} \mathrm{~cm}^{-1}$ at $280 \mathrm{~nm}$. Recombinant B. subtilis $\mathrm{MSDH}$ was produced and purified by ammonium sulfate fractionation, gel filtration, and anion exchange chromatography as described. ${ }^{37} \mathrm{MSDH}$ concentration was determined spectrophotometrically using a molar absorption coefficient of $2.04 \times$ $10^{5} \mathrm{M}^{-1} \mathrm{~cm}^{-1}$ at $280 \mathrm{~nm}$. In the text, both ALDH1A2 and $\mathrm{MSDH}$ concentrations are expressed per monomer (normality, $\mathrm{N})$.

Kinetic Parameters for Wild Type and Mutated ALDH1A2 under Steady-State Conditions. Initial rate measurements were carried out at $25{ }^{\circ} \mathrm{C}$ on a SAFAS UV mc2 spectrophotometer, by following the appearance of $\mathrm{NADH}$ at $340 \mathrm{~nm}$ in $20 \mathrm{mM}$ Hepes $\mathrm{pH} 8.5$ buffer containing $150 \mathrm{mM}$ $\mathrm{KCl}, 1 \mathrm{mM}$ EDTA, and $0.05 \%$ Tween 80 (standard buffer). Except for the G271A mutant, initial rate data were fit to the Michaelis-Menten equation using nonlinear least-squares regression analysis. For the G271A mutant, the reaction was also monitored by $\mathrm{NADH}$ fluorescence, more sensitive than absorbance, and initial rate data were fit to Michaelis-Menten model with excess substrate inhibition to determine the $k_{\text {cat }}$ $K_{\mathrm{M}}$, and $K_{\mathrm{i}}$ values. All $K_{\mathrm{M}}$ values for decanal and $\mathrm{NAD}^{+}$were determined at saturating concentrations of $\mathrm{NAD}^{+}$and decanal, respectively.

FRET Experiments. Fluorescence spectra were recorded on a SAFAS Xenius fluorimeter in $500 \mu \mathrm{L}$ cuvettes, using 10 $\mathrm{nm}$ slits and fixed enzyme concentration of $5 \mu \mathrm{N}$. The Trp to NADH FRET signal was recorded by exciting the enzyme/ NADH complex Trp residues at $295 \mathrm{~nm}$ and measuring the NADH fluorescence emission band between 400 and $550 \mathrm{~nm}$. Presteady-state kinetic analyses were carried out on a SX19MV-R stopped-flow apparatus (Applied PhotoPhysics), and collected data were analyzed using the Pro-data software package. Progress curves of Trp to NADH FRET were recorded at $25^{\circ} \mathrm{C}$ in standard buffer by following fluorescence emission using a $395 \mathrm{~nm}$ cutoff filter after excitation at $295 \mathrm{~nm}$. For ALDH1A2, one syringe was filled with the wild-type or G271A and $\mathrm{NAD}^{+}$, and the other contained decanal in the presence or absence of $0.5 \mu \mathrm{M} \mathrm{LDH}$ and $10 \mathrm{mM}$ pyruvate (final concentrations after mixing). The effect of viscosity was tested in similar experiments carried out in the presence of 0 and $20 \%$ glycerol in the buffer. For $\mathrm{MSDH}$, one syringe was filled with $\mathrm{NAD}^{+}$-preactivated enzyme ${ }^{20}$ and $\mathrm{NAD}^{+}$and the other contained propionaldehyde and CoA. Progress curves were fit according to a triphasic expression.

Targeted Molecular Dynamics Simulations. The conformational transition of the cofactor within the GAPN active site was simulated using the Targeted Molecular Dynamics (TMD) method implemented in AMBER 9 package. ${ }^{38}$ The starting and end-point models were derived from one monomer (chain A) of the high-resolution X-ray structures of GAPN/NADP ${ }^{+}$complex (PDB code 2euh) and E268A GAPN covalently bound to its substrate (thioacylenzyme intermediate) in complex with $\mathrm{NADPH}$ (PDB code $2 \mathrm{qe} 0$ ). Models were constructed using the Biopolymer module of Insight II software package (Accelrys, San Diego, U.S.A.) and Pymol. The starting conformation of the thioacylenzyme/ NADPH complex was constructed by extracting the coordinates of the enzyme and $\mathrm{NADP}^{+}$from the 2euh structure. The glyceraldehyde-3-phosphate (CG3) from the $2 \mathrm{qe} 0$ structure was then inserted into the model and linked covalently to the catalytic Cys302 thiol. The end-point conformation was constructed by extracting the coordinates of GAPN and NADPH from the $2 \mathrm{qe} 0$ structure, and the E268A mutation was reversed to the wild type.

The molecular all-atom $\mathrm{ff}^{39}$ and the general AMBER gaff ${ }^{40}$ force fields were used for the enzyme and the substrate, respectively. Parameters of the Amber parameter database ${ }^{41}$ were used for the NADPH. To obtain a neutral charge of the simulated system, the appropriate number of counterions $\left(\mathrm{Na}^{+}\right)$was included. The systems were then solvated with TIP3P water molecules, using the rectangular parallelepiped box with a minimum distance of $0.12 \mathrm{~nm}$ between the solute and the simulation box edge. Preparation of simulations consisted of 6 initial energy minimization cycles (1000 steps of steepest descent followed by 4000 steps of conjugate gradient methods) where atomic positions of the protein backbone were restrained using a harmonic potential. The force constant was progressively diminished along the energy minimization procedure until a final minimization step with only low constraints on backbone atoms of subunit interface residues within the tetramer (amino acid regions 119-144 and 465475). The minimization steps were then followed by a slow heating to $298 \mathrm{~K}$ under constant volume over a period of 180 ps, while maintaining constraints on NADPH, CG3, and protein backbone atoms. The system was then equilibrated for 80 ps under constant temperature $(298 \mathrm{~K})$ and pressure (1 bar) conditions via the Beredsen algorithm ${ }^{42}$ with a coupling constant of 0.2 ps for both parameters. Harmonic constraints were gradually removed along the equilibration phase, except for backbone atoms of residues 119-144 and 465-475. Longrange electrostatic forces were handled by the particle-mesh Ewald method. The time step of the simulations was $2.0 \mathrm{fs}$ and the SHAKE algorithm was used to constrain the lengths of all chemical bonds involving hydrogen atoms to their equilibrium values. $^{43}$

The TMD method allows observing large-scale conformational transitions between two known molecular conformations. This is accomplished by an additional steering force based on a mass-weighted RMSD with respect to reference target conformation that is applied in the force field as an extra harmonic potential energy term of the form

$$
E=0.5 k r N(\operatorname{RMSD}(t)-\operatorname{taRMSD}(t))^{2}
$$



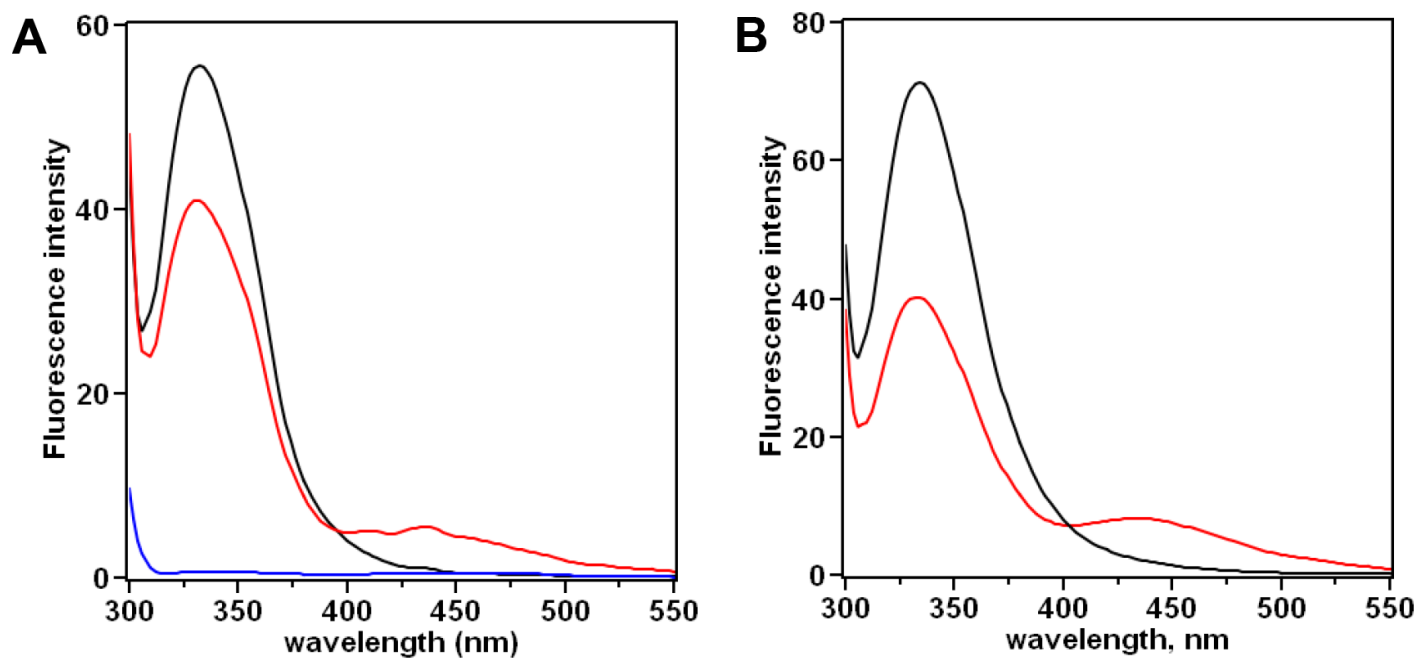

Figure 2. Trp to NMNH FRET signal within ALDH1A2/NADH complex. Fluorescence emission spectra of wild-type (A) and G271A (B) $\operatorname{ALDH1A} 2(5 \mu \mathrm{N})$ in the absence (black) and presence (red) of saturating NADH concentration (A, $5 \mu \mathrm{M}$ and $\mathrm{B}, 15 \mu \mathrm{M})$. The excitation wavelength was set at $295 \mathrm{~nm}$ to selectively excite Trp residues. The emission spectrum of free NADH $(5 \mu \mathrm{M})$ in the same conditions is shown in A (blue) for comparison.
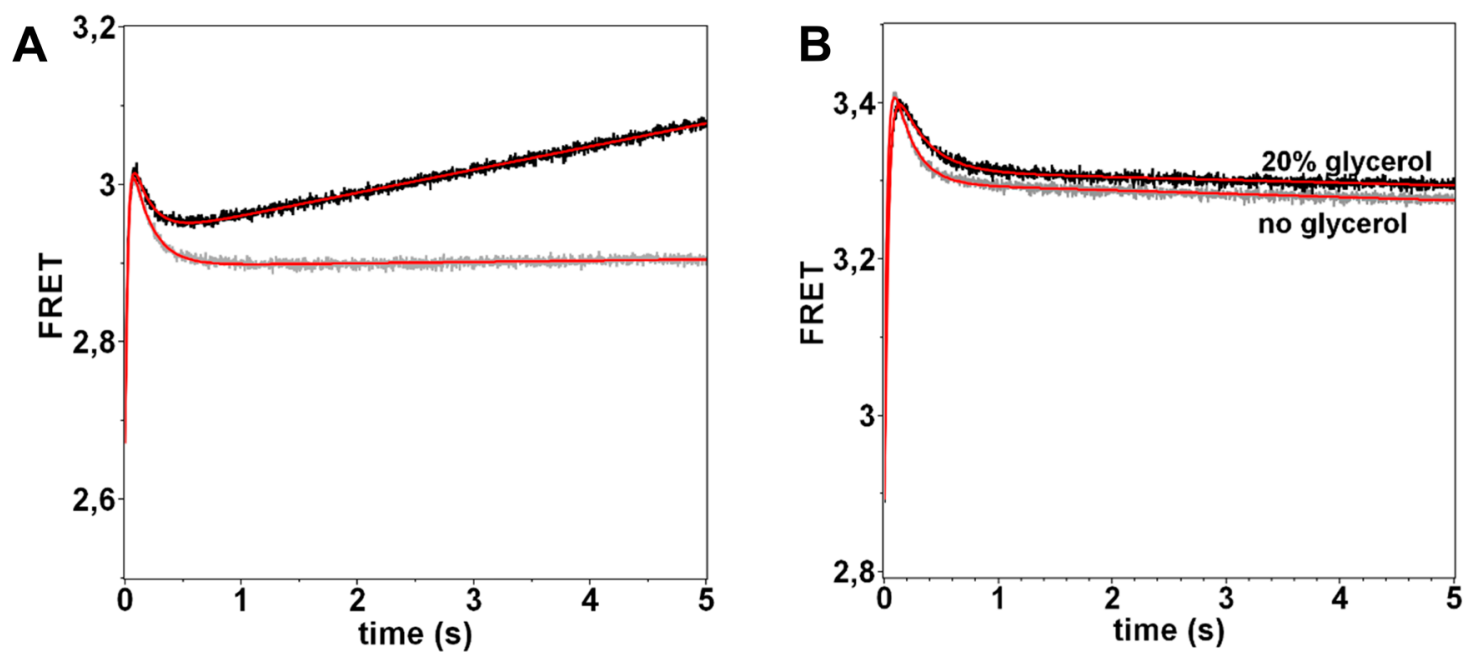

Figure 3. FRET-monitoring of the ALDH1A2-catalyzed reaction. (A) Single turnover kinetics of NADH production monitored by FRET for the wild-type ALDH1A2 $(1 \mu \mathrm{N})$ catalyzed reaction in the absence (black) and presence (gray) of the pyruvate/LDH coupled system. (B) Same experiment in the presence of LDH and with (black) or without (gray) 20\% glycerol. The biexponential fits are shown in red. Cofactor and substrate concentrations are $2 \mathrm{mM} \mathrm{NAD}^{+}$and $50 \mu \mathrm{M}$ decanal. The excitation wavelength is set at $295 \mathrm{~nm}$, and the fluorescence signal is monitored using a $395 \mathrm{~nm}$ cutoff filter to select $\mathrm{NADH}$ emission.

where $E$ is the biasing potential energy in $\mathrm{kcal} / \mathrm{mol}$ during the TMD simulation, $k r$ is the force constant, $N$ is the number of atoms, $\operatorname{RMSD}(t)$ is the root-mean-square deviation of the current structure at time $t$ relative to the target structure, and $\operatorname{taRMSD}(t)$ is the target RMSD value at time $t$. The tgtfitmask defines the atoms used for the rms superposition between the current structure and the reference structure and consists in atoms of all major secondary structures of the enzyme. The tgtrmsmask defines the atoms used for the rms difference calculation (and hence the restraint force) and includes all non-hydrogen atoms of the NADPH. The conformational transition from the initial to the final target geometry is driven by decreasing the value of the $\operatorname{RMSD}(t)$ to 0 as a function of the simulation time. A force constant of $0.01-0.6 \mathrm{kcal} \mathrm{mol}^{-1}$ $\AA^{-2}$ applied over $2 \mathrm{~ns}$ appeared sufficient to find a path for the transition. Altogether, 5 replicates of the TMD simulations were computed (Figure S4).
The resulting trajectories were analyzed using the Ptraj module of the AMBER 9 package. All graphics were prepared using Pymol ${ }^{44}$ (Schrodinger, Portland, OR, U.S.A.).

\section{RESULTS}

Kinetic Assessment of Cofactor Flip during Catalysis by Trp-NMNH FRET. To establish a methodology to assess cofactor flip, we considered the fluorescent properties of $\mathrm{NADH}$ conveyed by its $\mathrm{NMNH}$ moiety. Because Trp fluorescence emission spectrum overlaps the excitation spectrum of $\mathrm{NADH}$, we tested the hypothesis of a FRET between these fluorophores. Indeed, because FRET is highly sensitive to the distance and orientation between fluorophores, it can only occur for enzyme-bound $\mathrm{NADH}$ and thereby provides a conformation-sensitive probe of the NMNH moiety within the active site. First tests of selective excitation of GAPN Trp residues at $295 \mathrm{~nm}$ produced no significant signal 
Table 1. Steady State Kinetic Parameters of Wild-Type and ELGG Loop Mutant ALDH1A2 ${ }^{a}$

\begin{tabular}{|c|c|c|c|c|c|c|}
\hline \multirow[b]{2}{*}{ ALDH1A2 } & \multirow[b]{2}{*}{$k_{\mathrm{cat}}\left(\mathrm{s}^{-1}\right)$} & \multicolumn{2}{|c|}{$\mathrm{NAD}^{+}$} & \multicolumn{2}{|c|}{ decanal } & \multirow[b]{2}{*}{ rate-limiting step } \\
\hline & & $K_{\mathrm{M}}(\mu \mathrm{M})$ & $\overline{K_{i}(\mu \mathrm{M})}$ & $K_{\mathrm{M}}(\mu \mathrm{M})$ & $K_{i}(\mu \mathrm{M})$ & \\
\hline wild type ${ }^{b}$ & $2.2 \pm 0.2$ & $64 \pm 4$ & & $6 \pm 1$ & & deacylation \\
\hline G270A & $<0.001$ & & & & & acylation \\
\hline G271A & $0.029 \pm 0.003$ & $14 \pm 4$ & $1550 \pm 300$ & $3.2 \pm 1.1$ & $49 \pm 19$ & deacylation \\
\hline
\end{tabular}

${ }^{a}$ Parameters for $\mathrm{NAD}^{+}$and decanal were determined in the presence of a fixed concentration of decanal $(30 \mu \mathrm{M})$ and NAD $(100 \mu \mathrm{M})$, respectively. The G271A kinetics were fit against excess substrate inhibition model. The G271A rate-limiting step is deduced from single turnover experiment shown Figure 6. ${ }^{b}$ Data from ref 23.

corresponding to a FRET between the enzyme and NADPH. Conversely, in the presence of ALDH1A2 and NADH, a specific emission band centered at $436 \mathrm{~nm}$ similar to $\mathrm{NADH}$ emission was observed (Figure 2A). Concomitant decrease of the Trp emission band at $332 \mathrm{~nm}$ suggests transfer of Trp fluorescence to NADH, thus indicating a FRET signal.

The FRET signal was used to monitor NADH along the ALDH1A2 reaction under single turnover conditions, i.e., to observe the first cycle of catalysis. Using the $\mathrm{NADH}$ intrinsic fluorescence signal, we previously showed that the presteady state kinetics of decanal oxidation consist of a fast exponential burst of $\mathrm{NADH}$ production corresponding to hydride transfer, followed by a slower, linear phase corresponding to ratelimiting deacylation that reflects steady state kinetics. ${ }^{23}$ Using the FRET signal revealed a three-phase kinetic profile with a fast exponential burst characterized by a rate constant of $50 \mathrm{~s}^{-1}$ (see below) close to the value obtained using direct $\mathrm{NADH}$ fluorescence $\left(36 \mathrm{~s}^{-1}\right){ }^{23}$ thus attributed to hydride transfer (Figure 3A). A linear slow phase was also observed, likely produced by residual low signal of free $\mathrm{NADH}$ after release. In addition, reaction monitoring by FRET revealed a decreasing first order phase suggesting a change in the conformation of the $\mathrm{NMNH}$ moiety of $\mathrm{NADH}$ within the active site during the catalytic cycle. Adding the pyruvate/LDH coupled assay in excess to rapidly consume free $\mathrm{NADH}$ simplified the kinetics by silencing the linear phase and confirmed that the two exponential phases reflect events that take place within the active site (Figure 3A). Thus, the decreasing phase likely reflects $\mathrm{NMNH}$ moiety flipping within the thioacylenzyme active site, implying that the FRET signal intensity is lower for the flipped than for the hydride transfer conformation. Analysis of the biexponential kinetics quantified the rate constants of the burst and flip phases to 50 and $5.1 \mathrm{~s}^{-1}$, respectively. This assignment is further supported by the $30 \%$ decrease in the rate-constant associated with this kinetic phase upon addition of glycerol, a viscogen that is expected to slow down conformational transitions (Figure 3B). Similar results were obtained using saccharose as viscogen (not shown). The similarity between the steady state rate constant $\left(2.2 \mathrm{~s}^{-1}\right.$, Table 1) and the $5.1 \mathrm{~s}^{-1}$ phase revealed that the flip event is partially rate-limiting and that the thioacylenzyme/flipped $\mathrm{NADH}$ complex significantly accumulates during the steady state.

We next sought to identify the Trp residue(s) responsible for the kinetic detection of the NADH flip. ALDH1A2 contains six Trp residues (Figure S1). Because the FRET attribute was observed in rat ALDH1A2 (this study), human ALDH1A1 and ALDH2 $2^{32}$ but not GAPN, we substituted the four Trp conserved in the rat and human enzymes by Phe. All mutants were active (Table S1), and all but W168F showed a presteady state burst, implying acylation was not rate limiting (Figures 4 and S2). The W135F, W177F, and W452F mutants produced FRET signals with overall reduced amplitude, in

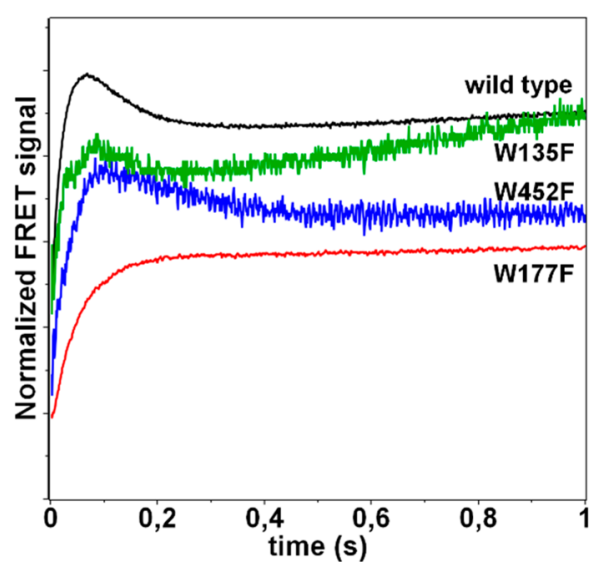

Figure 4. FRET-monitoring of the reaction catalyzed by wild-type and mutated ALDH1A2. Single turnover kinetics of NADH production monitored by FRET for wild-type and mutant ALDH1A2 $(1 \mu \mathrm{N})$ in the presence of $\operatorname{NAD}^{+}(1 \mathrm{mM})$ and decanal $(50 \mu \mathrm{M})$ under the same conditions as in Figure 3. The fluorescence traces of the mutants are offset and normalized according to the wild type.

accordance with the removal of a Trp residue. However, while wild-type, W452F, and W135F ALDH1A2 oxidized decanal by following three-phase kinetics, no decreasing kinetic phase was observed for the W177F mutant, suggesting that W177 acts as the major flip-sensitive fluorescent probe (Figure 4). Accordingly, the distances between Trp177 CE2 atom from ALDH1A2-NAD ${ }^{+}$complex structure (1bi9), superimposed on pre- and postflip GAPN structures (2euh and $2 \mathrm{qe} 0$, respectively), and $\operatorname{NADP}(\mathrm{H})$ nicotinamide $\mathrm{C} 4$ atom, changes from 8.3 to $19.5 \AA$, respectively (Figure S3).

Targeted Molecular Dynamics Simulations Identify $E^{268}$ LGG $^{271}$ Active Site Loop As a Molecular Determinant of Cofactor Flipping. While the starting and endpoints of cofactor flip have been trapped and characterized by $\mathrm{X}$-ray crystallography, neither the conformational transition pathway nor the driving force allowing this movement are known. Previous crystal structures comparison pointed out that no significant conformational change of the protein structure seems to be required for the NMNH movement to occur. ${ }^{34} \mathrm{~A}$ rotation of the PN-O5D-C5D-C4D dihedral bond connecting the ribose $\mathrm{C} 4-\mathrm{C} 5$ atoms to the $\mathrm{O}-\mathrm{P}$ atoms of the NMN moiety (from $+79.1^{\circ}$ to $-126.9^{\circ}$ ) is observed when comparing the two structures, and the NMNH was found to move away from the thioacyl intermediate (up to $10 \AA$ ), while the adenine moiety remains bound in the same pocket.

In this work, our objective was not to perform an in-depth and exhaustive computational investigation of the system but rather to propose mechanistic hypotheses regarding the molecular features that could play a role on the cofactor 


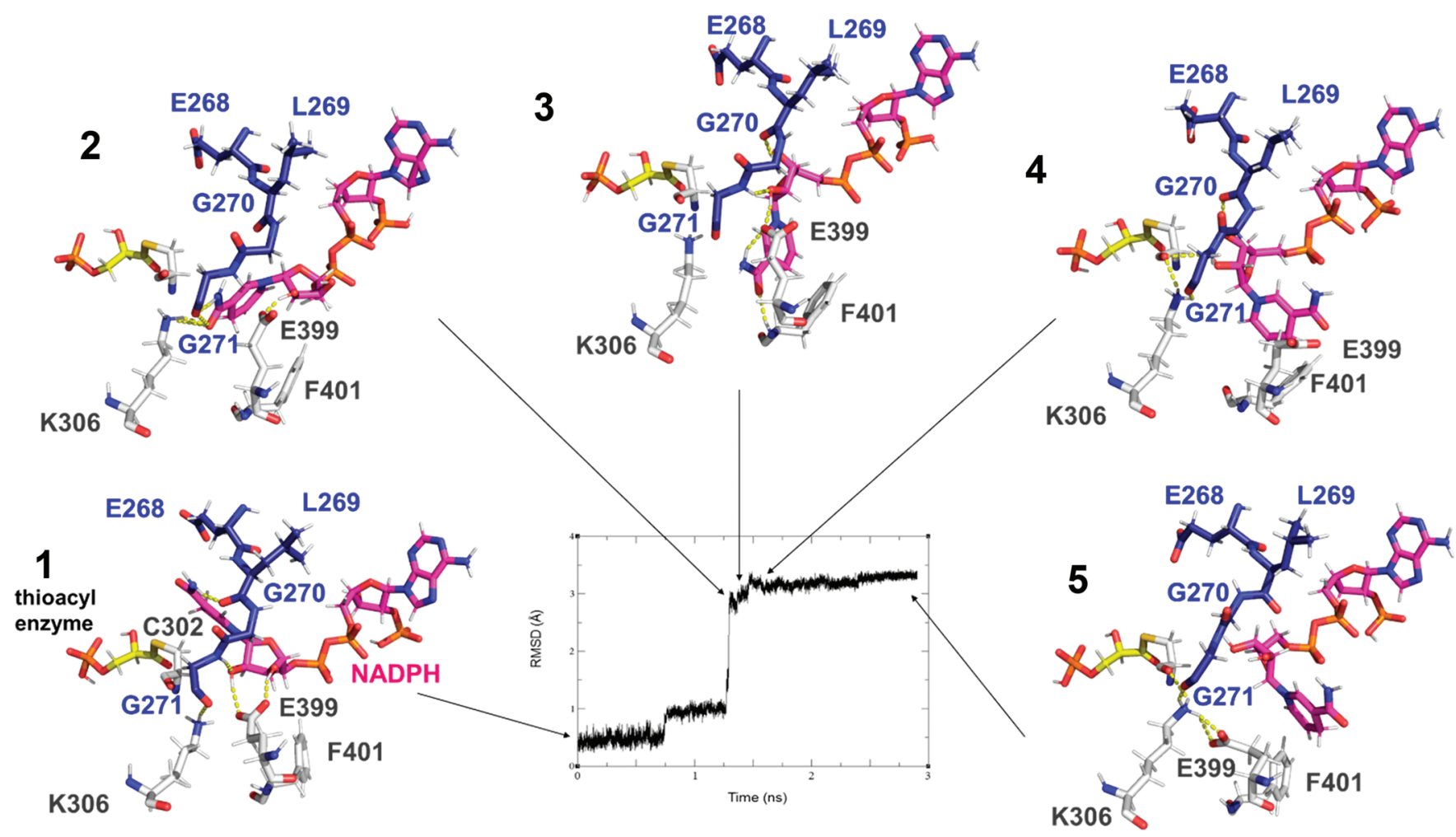

Figure 5. Snapshots ( 1 to 5 ) of the modeled dynamics showing the movements of the cofactor in the active site environment along the TMD simulation. The ELGG loop carbon atoms are colored in blue, NADPH in magenta, and the substrate linked as a thioacylenzyme is yellow. Hbonds are shown are yellow (dash). The graph shows the cofactor RMSD along the TMD simulation.

flipping. Thus, to identify the molecular features that control the flip, we used TMD simulations to characterize the cofactor conformational transition using the available crystal structures of GAPN in which the NMN moiety of the cofactor is defined in distinct conformations. We used as a starting point the holo GAPN structure (PDB code 2euh), which incorporates $\mathrm{NADP}^{+}$in an extended conformation. The C302S-NADP ${ }^{+}-$ G3P ternary complex (PDB code 1qi1) was excluded as an alternative, because the cofactor is retracted and the substrate shifted compared to its position in the thioacylenzyme complex. The thioacylenzyme/NADPH complex (PDB code $2 \mathrm{qe} 0$ ) gave the ending point with the NADPH in the flipped conformation. $^{34,35}$

The five replicates of TMD simulations revealed a similar plausible pathway for the cofactor flip (Figures 5 and S4 and Movie S1). For clarity purpose, we chose to describe in details only one of the TMD simulations, representative of the conformational transition (Figure 5 and Movie S1). The overall RMSD between the initial and the target structures of the cofactor was $\sim 3 \AA$. The transition was achieved within the first $2 \mathrm{~ns}$, and the system remained in the target conformation (PDB code $2 \mathrm{qe} 0$ ) for the rest of the simulation. Before the flip, in addition to Glu399 carboxylate interactions with the ribose $2^{\prime}$ - and $3^{\prime}-\mathrm{OH}, \mathrm{NMNH}$ is stabilized through hydrogen bonds between the backbone carbonyl of Leu269 and the nicotinamide $\mathrm{NH}_{2}$ group, and between the amide of Gly271 and the ribose $2^{\prime}-\mathrm{OH}$ (Figure 5 step 1 ). Over the first $\sim 0.75$ ns, our results suggest a conformational pathway involving local fluctuations of the nicotinamide which result in loss of the $\mathrm{H}$-bond between the nicotinamide $\mathrm{NH}_{2}$ and Leu269 carbonyl oxygen (Figure 5, step 2). As a result, the $\mathrm{E}^{268} \mathrm{LGG}^{271}$ loop connecting the $\beta 11$ and $\beta 12$ strands becomes more flexible.
After a first plateau between 0.75 and $1.25 \mathrm{~ns}$, a drastic transition is observed on the RMSD profile, corresponding to the NMNH moiety switch which is accompanied by tangible conformational changes within the $\mathrm{E}^{268} \mathrm{LGG}^{271}$. This loop, highly conserved in hydrolytic ALDHs, appears as a potentially critical element that has to move away to avoid a steric clash between the moving NMNH moiety and the Leu269-Gly270 peptide bond (Figure 5 steps 2-3). Of note, the presence of adjacent Gly270-Gly271 should provide flexibility to the loop, allowing NMNH switch while limiting unfavorable steric contacts.

Upon progression of NMNH toward the flipped conformation, the $\mathrm{E}^{268} \mathrm{LGG}^{271}$ backbone shifts back to a position very similar to its initial conformation (Figures 5, steps 4-5, and S5 and Movie S1). In addition, the conformational transition of the NMNH moiety is accompanied by a progressive reorganization of the interactions between GAPN (in particular the ELGG loop and invariant Glu399) and the NMNH moiety (Figure 5). All together these data allowed us to provide structural context into how NMNH can experience conformational change between the two states with a modest energy barrier given the low force constant (lower than $0.6 \mathrm{kcal}$ $\mathrm{mol}^{-1} \AA^{-2}$ ) required to drive the conformational transition using TMD.

Cofactor Flip Relies on ELGG Loop Flexibility. We next inferred that the potential role of the $\mathrm{E}^{268} \mathrm{LGG}^{271}$ loop in $\mathrm{NMNH}$ flipping should be linked to the flexibility provided by the invariant Gly residues. Substitution of Gly270 by Ala decreased drastically ALDH1A2 activity below the detection threshold (Table 1). This effect could result from decrease of loop flexibility and steric clash between Ala270 side chain and NMN leading to alteration of nicotinamide positioning that 
impairs hydride transfer. Substituting Gly271 by Ala significantly decreased the maximum observed steady state rate constant by 2 orders of magnitude $\left(0.029 \mathrm{vs} 2.2 \mathrm{~s}^{-1}\right.$, Table $1)$. As for the wild type, selective excitation of ALDH1A2 Trp residues at $295 \mathrm{~nm}$ in the presence of NADH resulted in a specific emission band at $434 \mathrm{~nm}$ that indicated Trp to NADH FRET (Figure 2B). This allowed single turnover kinetics of the G271A mutant to be monitored, which revealed a three-phase kinetic profile as for the wild-type, with a burst phase showing that acylation remained non rate-limiting (Figure 6).

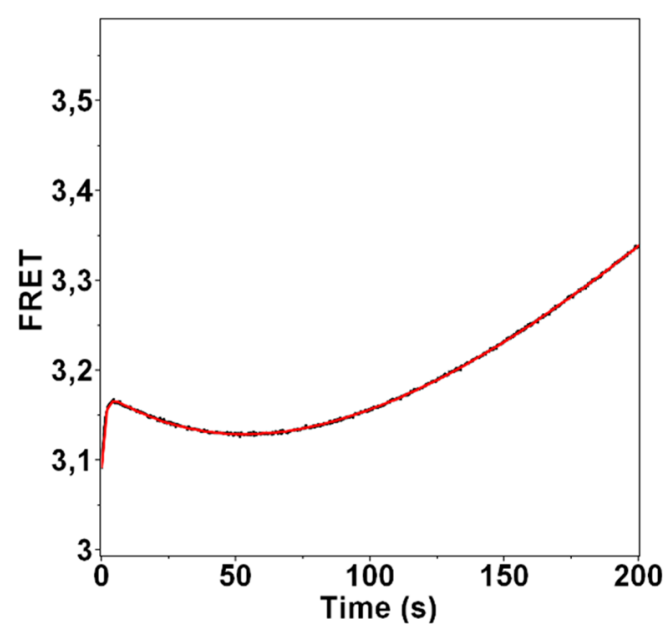

Figure 6. Single turnover kinetics of NADH production monitored by FRET for the G271A ALDH1A2. The experiment was performed under conditions similar to Figure 3 except for enzyme concentration $(2.5 \mu \mathrm{N})$. The biexponential plus slope fit is shown in red. $\mathrm{NAD}^{+}$and decanal concentrations are 100 and $20 \mu \mathrm{M}$, respectively. The excitation wavelength is set at $295 \mathrm{~nm}$, and the fluorescence signal is monitored using a $395 \mathrm{~nm}$ cutoff filter to select NADH emission.

Accordingly, the fast-increasing phase followed by decreasing and linear phases were attributed to the hydride transfer (rate constant $1.0 \pm 0.03 \mathrm{~s}^{-1}$ ), NMNH flip (rate constant $0.010 \pm$ $\left.0.001 \mathrm{~s}^{-1}\right)$, and deacylation events, respectively. Simplification of the kinetics using the $\mathrm{LDH} /$ pyruvate coupled assay was not successful in this case due to large background signal generated by the coupled system on this longer time scale. Nevertheless, the 500 -fold decrease of the flip rate constant from 5.1 to 0.010 $\mathrm{s}^{-1}$ indicated that the G271A mutation drastically impairs the $\mathrm{NMNH}$ movement during catalysis. Compared to the steady state rate constant of $0.029 \mathrm{~s}^{-1}$, the flip rate constant falling to the same order of magnitude suggests that NMNH movement becomes rate-limiting for the overall reaction. This confirms the critical role of the $\mathrm{E}^{268} \mathrm{LGG}^{271}$ loop mobility to allow efficient NMNH flip and catalysis by ALDH1A2.

Cofactor Flip Extends to CoA-Dependent Aldehyde Dehydrogenases. To evaluate the existence of a flip event in CoA-dependent ALDH catalysis, we tested the FRET properties of $B$. subtilis MSDH. As for ALDH1A2, selective excitation of $\mathrm{MSDH}$ at $295 \mathrm{~nm}$ in the presence of $\mathrm{NADH}$ resulted in a specific emission band at $434 \mathrm{~nm}$ that indicated Trp to NADH FRET (Figure 7A). To favor visualization of a kinetic signal associated with a flip event by FRET, we used a substrate analogue (propionaldehyde) and low CoA concentration to slow down the acylation and deacylation phases compared to the natural substrate, respectively. ${ }^{45}$ Single turnover kinetics of the $\mathrm{MSDH}$ reaction in these conditions revealed a three-phase kinetic profile similar to ALDH1A2, with a burst phase corresponding to acylation (rate constant $135 \pm 6 \mathrm{~s}^{-1}$ ), followed by a decreasing first order phase characterized by a rate constant of $39 \pm 2 \mathrm{~s}^{-1}$, suggesting $\mathrm{NMNH}$ flipping (Figure 7B). To confirm that this signal resulted from a flip event as for hydrolytic ALDH1A2, we tested whether it depended on the same Trp177 probe. The kinetic profile of the W177F MSDH-catalyzed reaction shows altered relative amplitudes of the three phases, with a marked decrease of the second phase (Figure 7B), indicating a significant contribution of the Trp177 to this signal. Therefore, the NMNH flip process is also an integral event of the catalytic pathway of MSDH.
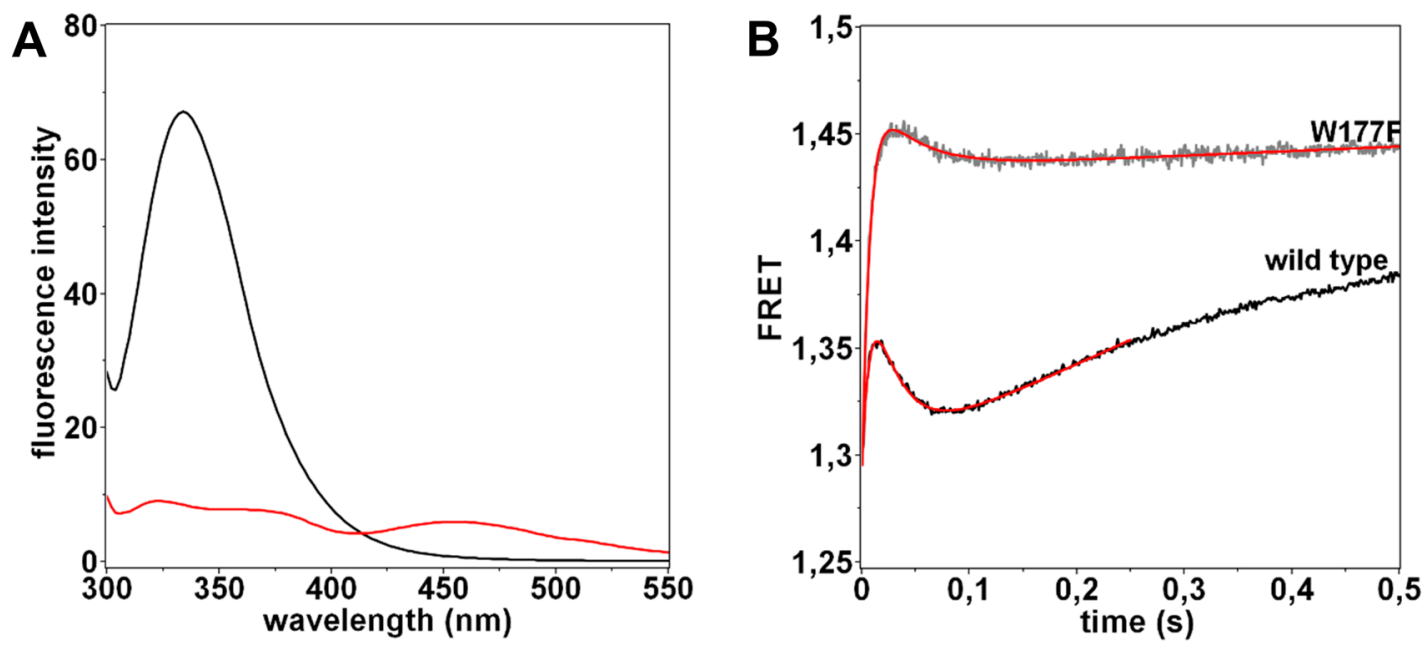

Figure 7. FRET-monitoring of the cofactor flip in the CoA-dependent MSDH-catalyzed reaction. (A) Fluorescence emission spectra of MSDH (5 $\mu \mathrm{N})$ in the absence (black) and presence (red) of saturating NADH concentration $(200 \mu \mathrm{M})$. The fluorescence emission band of Trp at $332 \mathrm{~nm}$ is bleached by the screen effect due to high NADH concentration. The excitation wavelength is set at $295 \mathrm{~nm}$ to selectively excite Trp residues. (B) Single turnover kinetics of NADH production monitored by FRET for wild-type (black) and W177F (gray) MSDH (16 $\mu$ N). The biexponential plus slope fits are shown in red. Concentrations are $2 \mathrm{mM} \mathrm{NAD}^{+}, 50 \mu \mathrm{M} \mathrm{CoA}$ and $500 \mathrm{mM}$ propionaldehyde. The excitation wavelength is set at $295 \mathrm{~nm}$, and the fluorescence signal is monitored using a $395 \mathrm{~nm}$ cutoff filter to select NADH emission. 


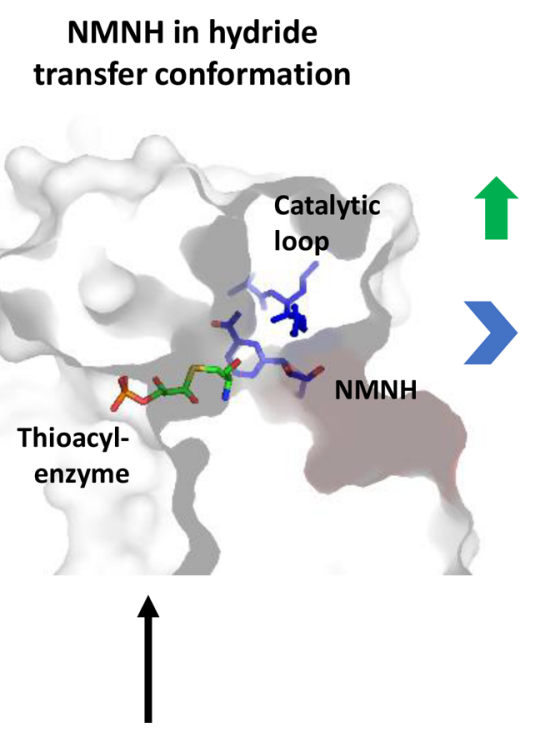

ACYLATION
FLIP

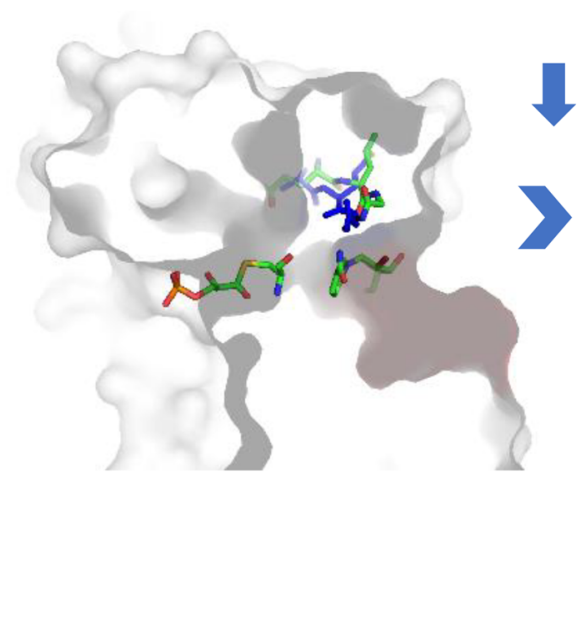

NMNH in exit pocket

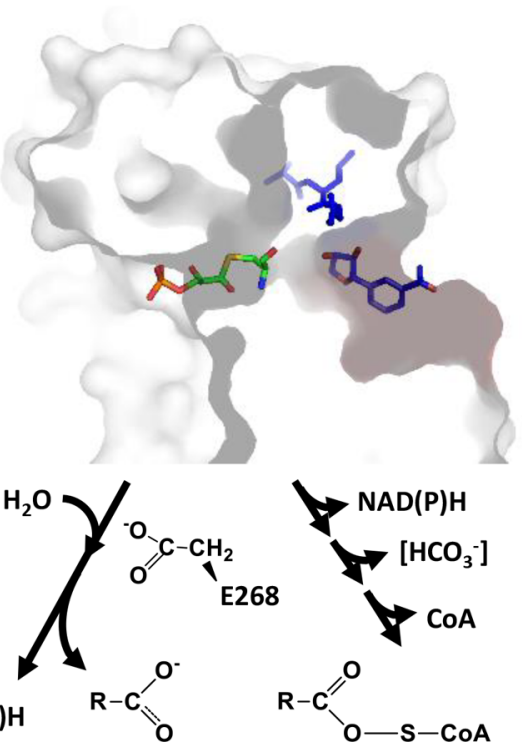

Figure 8. Active site dynamics in ALDH deacylation mechanism.

\section{DISCUSSION}

In the ALDH catalytic cycle, structural data showed that completion of the deacylation requires a movement of the reduced NMN moiety of the cofactor. ${ }^{34,35}$ Combined strategy using a Trp to NMNH FRET signal to monitor the NMNH conformational motions within the active site and TMD simulations revealed the critical role of a four-residue catalytic loop as a gatekeeper of cofactor flip, a property common to hydrolytic and CoA-dependent ALDHs.

One key chicken-and-egg question relates to the driving force that promotes this isomerization, in other words does reduction of the cofactor or catalytic loop intrinsic flexibility trigger the flip? In hydrolytic ALDHs, by interaction with Thr244 $\beta$-methyl group, the NMN ring initially adopts a metastable conformation catalytically competent for hydride transfer (Figure 1C). Moreover, the oxidized nicotinamide moiety is also able to flip, ${ }^{33}$ suggesting that it is not a redoxcontrolled motion and that both conformations are energetically close, as supported by TMD simulations, with an energy barrier below $0.6 \mathrm{kcal} \mathrm{mol}^{-1} \AA^{-2}$. Thus, it is tempting to speculate that, owing to its flexibility, the catalytic loop updown shift enables the NMNH to reach the exit pocket by concerted reorganization of the $\mathrm{H}$-bond network (Figure 5). In support of this hypothesis, reducing the $\mathrm{E}^{268} \mathrm{LGG}^{271}$ loop flexibility in ALDH1A2 by the G271A mutation drastically impaired the flip efficiency, thereby reducing accordingly the steady state rate constant of the overall reaction.

Is cofactor flip a general feature common to all ALDHs? Observation of a similar kinetic event for the CoA-dependent MSDH suggest that it is indeed the case, although its mechanistic relevance remains to be established. Indeed, $\mathrm{NADH}$ flip should not be required for transthioesterification due to distinct $\mathrm{CoA}$ and $\mathrm{NAD}^{+}$binding sites, ${ }^{45,46}$ thus excluding direct steric hindrance. We propose that $\mathrm{NMNH}$ movement makes room for the decarboxylated thioacylenzyme to adopt a position suitable for an efficient CoA attack. Besides, we showed previously that the pocket that is postulated to constitute the exit door of the reduced cofactor in hydrolytic
ALDHs is conserved in MSDH, thus suggesting a similar dissociation pathway. Furthermore, hydrolytic and CoAdependent ALDHs adopt superimposable folds, including the active site and the 268-271 gatekeeper catalytic loop (Figure S3). Interestingly, while the loop sequence is strictly conserved in hydrolytic ALDHs, in particular for the 268 position that requires a catalytic Glu for deacylation, it is more variable within the CoA-dependent family. However, the invariant Gly270 and Ala271 provide the required flexibility to allow the flip event in $\mathrm{MSDH}^{47}$ Irrespective of the loop sequence, the flip rate constants range from 5 to $39 \mathrm{~s}^{-1}$ for ALDH1A2 and MSDH, respectively, and reaches at least $60 \mathrm{~s}^{-1}$ for GAPN (i.e., the $k_{\text {cat }}$ value), which indicates that the catalytic loop controls the NMNH motion but not its kinetics.

By combining our past and present kinetic and structural studies with molecular dynamics simulations, ${ }^{17,24,34}$ it is now possible to build up an integrated scenario of the sequence of events encompassed in deacylation (Figure 8). As soon as hydride transfer occurs, the NMNH is allowed to flip through concerted up and down motion of the gatekeeper loop and reaches the hydrophobic exit pocket. In hydrolytic ALDHs, this makes the hydrolysis possible by enabling the Glu268 to activate the water molecule, while in their CoA-dependent counterparts it possibly contributes to make the thioacylenzyme competent for transthioesterification. In MSDH, weaker stabilization of the adenine ring triggers early $\mathrm{NADH}$ release ${ }^{24}$ followed by the $\beta$-decarboxylation process, ${ }^{20}$ subsequent CoA nucleophilic attack and product release. Except for the decarboxylation, this scenario may hold for all CoA-dependent ALDHs. In hydrolytic ALDHs, cofactor stabilization mainly results from interactions with adenine moiety, ${ }^{24}$ thus explaining the late release of $\mathrm{NAD}(\mathrm{P}) \mathrm{H}$, which occurs after hydrolysis and product dissociation. In conclusion, our work provides significant insight into ALDHs catalytic mechanism in bridging the gap between the well-studied chemical steps and a conformational transition essential for catalysis. 


\section{ASSOCIATED CONTENT}

\section{S Supporting Information}

The Supporting Information is available free of charge on the ACS Publications website at DOI: 10.1021/acscatal.8b03951.

Structure-based alignment of ALDHs sequences (Figure S1); non-normalized FRET-monitoring of the reaction catalyzed by wild-type and mutated ALDH1A2 (Figure S2); steady state kinetic parameters of wild-type and Trp mutant ALDH1A2 (Table S1); superimposition of the 268-271 catalytic loop of GAPN, ALDH1A2, and MSDH (Figure S3); RMSD profile of heavy atoms of the NADP cofactor for 5 replicates of TMD simulations (Figure S4); analysis of $\mathrm{E}^{268} \mathrm{LGG}^{271}$ loop flexibility along TMD simulation (Figure S5) (PDF)

Conformational transition of the NADPH cofactor within the GAPN active site along the TMD simulation (AVI)

\section{AUTHOR INFORMATION}

\section{Corresponding Authors}

*E-mail: sophie.rahuel@univ-lorraine.fr.

*E-mail: francois.talfournier@univ-lorraine.fr.

\section{ORCID}

Sophie Rahuel-Clermont: 0000-0001-5143-0105

Isabelle André: 0000-0001-6280-4109

\section{Present Address}

${ }^{\S}$ UMR 1136 Interactions Arbres/Microorganismes, Faculté des Sciences et Technologies, Université de Lorraine/INRA, Vandoeuvre-lès-Nancy, France.

\section{Notes}

The authors declare no competing financial interest.

\section{ACKNOWLEDGMENTS}

We gratefully acknowledge J. Charbonnel for very efficient technical help. R.B. was supported by the French Research Ministry. Authors would like to thank J. Esque for his help with Steered and Targeted MD simulations and discussion of results. This work was supported in part by CNRS, the University de Lorraine and local funds from the Région Grand Est. This work was granted access to the Biophysics and structural biology platform of UMS2008 IBSLor CNRS-ULINSERM and to the Computing mesocenter of Région MidiPyrénées (CALMIP, Toulouse, France).

\section{ABBREVIATIONS}

ALDH,aldehyde dehydrogenase; FRET,fluorescence resonance energy transfer; GAPN,nonphosphorylating glyceraldehyde 3phosphate dehydrogenase; LDH,lactate dehydrogenase; MSDH,methylmalonate semialdehyde dehydrogenase; NMN, $\mathrm{NMNH}$,nicotinamide mononucleotide oxidized and reduced forms; TMD,targeted molecular dynamics simulation.

\section{REFERENCES}

(1) Pudney, C. R.; Heyes, D. J.; Khara, B.; Hay, S.; Rigby, S. E. J.; Scrutton, N. S. Kinetic and Spectroscopic Probes of Motions and Catalysis in the Cytochrome P450 Reductase Family of Enzymes: Electron Transfer in Cytochrome P450 Reductase. FEBS J. 2012, 279, $1534-1544$

(2) Narayanan, C.; Bernard, D. N.; Doucet, N. Role of Conformational Motions in Enzyme Function: Selected Methodologies and Case Studies. Catalysts 2016, 6, 81 .
(3) Nashine, V. C.; Hammes-Schiffer, S.; Benkovic, S. J. Coupled Motions in Enzyme Catalysis. Curr. Opin. Chem. Biol. 2010, 14, 644651.

(4) Eisenmesser, E. Z.; Bosco, D. A.; Akke, M.; Kern, D. Enzyme Dynamics During Catalysis. Science 2002, 295, 1520-1523.

(5) Zeng, T.; Leimkühler, S.; Wollenberger, U.; Fourmond, V. Transient Catalytic Voltammetry of Sulfite Oxidase Reveals Rate Limiting Conformational Changes. J. Am. Chem. Soc. 2017, 139, 11559-11567.

(6) Gagné, D.; Doucet, N. Structural and Functional Importance of Local and Global Conformational Fluctuations in the RNase A Superfamily. FEBS J. 2013, 280, 5596-5607.

(7) Champion, E.; Guérin, F.; Moulis, C.; Barbe, S.; Tran, T. H.; Morel, S.; Descroix, K.; Monsan, P.; Mourey, L.; Mulard, L. A.; Tranier, S.; Remaud-Simeon, M.; André, I. Applying Pairwise Combinations of Amino Acid Mutations for Sorting Out Highly Efficient Glucosylation Tools for Chemo-Enzymatic Synthesis of Bacterial Oligosaccharides. J. Am. Chem. Soc. 2012, 134, 1867718688.

(8) Desamero, R.; Rozovsky, S.; Zhadin, N.; McDermott, A.; Callender, R. Active Site Loop Motion in Triosephosphate Isomerase: T-Jump Relaxation Spectroscopy of Thermal Activation. Biochemistry 2003, 42, 2941-2951.

(9) Zhadin, N.; Gulotta, M.; Callender, R. Probing the Role of Dynamics in Hydride Transfer Catalyzed by Lactate Dehydrogenase. Biophys. J. 2008, 95, 1974-1984.

(10) Henzler-Wildman, K.; Kern, D. Dynamic Personalities of Proteins. Nature 2007, 450, 964-972.

(11) Marchitti, S. A.; Brocker, C.; Stagos, D.; Vasiliou, V. Non-P450 Aldehyde Oxidizing Enzymes: The Aldehyde Dehydrogenase Superfamily. Expert Opin. Drug Metab. Toxicol. 2008, 4, 697-720.

(12) Ginestier, C.; Hur, M. H.; Charafe-Jauffret, E.; Monville, F.; Dutcher, J.; Brown, M.; Jacquemier, J.; Viens, P.; Kleer, C. G.; Liu, S.; Schott, A.; Hayes, D.; Birnbaum, D.; Wicha, M. S.; Dontu, G. ALDH1 Is a Marker of Normal and Malignant Human Mammary Stem Cells and a Predictor of Poor Clinical Outcome. Cell Stem Cell 2007, 1, $555-567$.

(13) Huang, E. H.; Hynes, M. J.; Zhang, T.; Ginestier, C.; Dontu, G.; Appelman, H.; Fields, J. Z.; Wicha, M. S.; Boman, B. M. Aldehyde Dehydrogenase 1 Is a Marker for Normal and Malignant Human Colonic Stem Cells (SC) and Tracks SC Overpopulation during Colon Tumorigenesis. Cancer Res. 2009, 69, 3382-3389.

(14) Wan, C.; Shi, Y.; Zhao, X.; Tang, W.; Zhang, M.; Ji, B.; Zhu, H.; $\mathrm{Xu}$, Y.; Li, H.; Feng, G.; Lin, H. Positive Association between ALDH1A2 and Schizophrenia in the Chinese Population. Prog. NeuroPsychopharmacol. Biol. Psychiatry 2009, 33, 1491-1495.

(15) Kim, H.; Lapointe, J.; Kaygusuz, G.; Ong, D. E.; Li, C.; van de Rijn, M.; Brooks, J. D.; Pollack, J. R. The Retinoic Acid Synthesis Gene ALDH1a2 Is a Candidate Tumor Suppressor in Prostate Cancer. Cancer Res. 2005, 65, 8118-8124.

(16) Sheikh, S.; Ni, L.; Hurley, T. D.; Weiner, H. The Potential Roles of the Conserved Amino Acids in Human Liver Mitochondrial Aldehyde Dehydrogenase. J. Biol. Chem. 1997, 272, 18817-18822.

(17) Marchal, S.; Rahuel-Clermont, S.; Branlant, G. Role of Glutamate-268 in the Catalytic Mechanism of Nonphosphorylating Glyceraldehyde-3-Phosphate Dehydrogenase from Streptococcus Mutans. Biochemistry 2000, 39, 3327-3335.

(18) Shone, C. C.; Fromm, H. J. Steady-State and Pre-Steady-State Kinetics of Coenzyme A Linked Aldehyde Dehydrogenase from Escherichia Coli. Biochemistry 1981, 20, 7494-7501.

(19) Sohling, B.; Gottschalk, G. Purification and Characterization of a Coenzyme-A-Dependent Succinate-Semialdehyde Dehydrogenase from Clostridium Kluyveri. Eur. J. Biochem. 1993, 212, 121-127.

(20) Stines-Chaumeil, C.; Talfournier, F.; Branlant, G. Mechanistic Characterization of the MSDH (Methylmalonate Semialdehyde Dehydrogenase) from Bacillus Subtilis. Biochem. J. 2006, 395, 107115 .

(21) Rahuel-Clermont, S.; Arutyunov, D.; Marchal, S.; Orlov, V.; Muronetz, V.; Branlant, G. Thermal Destabilization of Non- 
Phosphorylating Glyceraldehyde-3-Phosphate Dehydrogenase from Streptococcus Mutans upon Phosphate Binding in the Active Site. J. Biol. Chem. 2005, 280, 18590-18597.

(22) Bordelon, T.; Montegudo, S. K.; Pakhomova, S.; Oldham, M. L.; Newcomer, M. E. A Disorder to Order Transition Accompanies Catalysis in Retinaldehyde Dehydrogenase Type II. J. Biol. Chem. 2004, 279, 43085-43091.

(23) Bchini, R.; Vasiliou, V.; Branlant, G.; Talfournier, F.; RahuelClermont, S. Retinoic Acid Biosynthesis Catalyzed by Retinal Dehydrogenases Relies on a Rate-Limiting Conformational Transition Associated with Substrate Recognition. Chem.-Biol. Interact. 2013, $202,78-84$

(24) Bchini, R.; Dubourg-Gerecke, H.; Rahuel-Clermont, S.; Aubry, A.; Branlant, G.; Didierjean, C.; Talfournier, F. Adenine Binding Mode Is a Key Factor in Triggering the Early Release of NADH in Coenzyme A-Dependent Methylmalonate Semialdehyde Dehydrogenase. J. Biol. Chem. 2012, 287, 31095-31103.

(25) Muñoz-Clares, R. A.; González-Segura, L.; Díaz-Sánchez, Á. G. Crystallographic Evidence for Active-Site Dynamics in the Hydrolytic Aldehyde Dehydrogenases. Implications for the Deacylation Step of the Catalyzed Reaction. Chem.-Biol. Interact. 2011, 191, 137-146.

(26) Gonnella, T. P.; Keating, J. M.; Kjemhus, J. A.; Picklo, M. J.; Biggane, J. P. Fluorescence Lifetime Analysis and Effect of Magnesium Ions on Binding of NADH to Human Aldehyde Dehydrogenase 1. Chem.-Biol. Interact. 2013, 202, 85-90.

(27) Perez-Miller, S. J.; Hurley, T. D. Coenzyme Isomerization Is Integral to Catalysis in Aldehyde Dehydrogenase. Biochemistry 2003, 42, 7100-7109.

(28) Blackwell, L. F.; Motion, R. L.; MacGibbon, A. K.; Hardman, M. J.; Buckley, P. D. Evidence That the Slow Conformation Change Controlling NADH Release from the Enzyme Is Rate-Limiting during the Oxidation of Propionaldehyde by Aldehyde Dehydrogenase. Biochem. J. 1987, 242, 803-808.

(29) Liu, Z. J.; Sun, Y. J.; Rose, J.; Chung, Y. J.; Hsiao, C. D.; Chang, W. R.; Kuo, I.; Perozich, J.; Lindahl, R.; Hempel, J.; Wang, B. C. The First Structure of an Aldehyde Dehydrogenase Reveals Novel Interactions between NAD and the Rossmann Fold. Nat. Struct. Biol. 1997, 4, 317-326.

(30) Cobessi, D.; Tete-Favier, F.; Marchal, S.; Azza, S.; Branlant, G.; Aubry, A. Apo and Holo Crystal Structures of an NADP-Dependent Aldehyde Dehydrogenase from Streptococcus Mutans. J. Mol. Biol. 1999, 290, 161-173.

(31) Johansson, K.; El-Ahmad, M.; Ramaswamy, S.; Hjelmqvist, L.; Jornvall, H.; Eklund, H. Structure of Betaine Aldehyde Dehydrogenase at 2.1 A Resolution. Protein Sci. 1998, 7, 2106-2117.

(32) Hammen, P. K.; Allali-Hassani, A.; Hallenga, K.; Hurley, T. D.; Weiner, H. Multiple Conformations of NAD and NADH When Bound to Human Cytosolic and Mitochondrial Aldehyde Dehydrogenase. Biochemistry 2002, 41, 7156-7168.

(33) Pailot, A.; D'Ambrosio, K.; Corbier, C.; Talfournier, F.; Branlant, G. Invariant Thr244 Is Essential for the Efficient Acylation Step of the Non-Phosphorylating Glyceraldehyde-3-Phosphate Dehydrogenase from Streptococcus Mutans. Biochem. J. 2006, 400, 521-530.

(34) D’Ambrosio, K.; Pailot, A.; Talfournier, F.; Didierjean, C.; Benedetti, E.; Aubry, A.; Branlant, G.; Corbier, C. The First Crystal Structure of a Thioacylenzyme Intermediate in the ALDH Family: New Coenzyme Conformation and Relevance to Catalysis. Biochemistry 2006, 45, 2978-2986.

(35) D’Ambrosio, K.; Pailot, A.; Talfournier, F.; Didierjean, C.; Benedetti, E.; Aubry, A.; Branlant, G.; Corbier, C. The First Crystal Structure of a Thioacylenzyme Intermediate in the ALDH Family: New Coenzyme Conformation and Relevance to Catalysis. Biochemistry 2007, 46, 10234-10235.

(36) Studier, F. W. Protein Production by Auto-Induction in High Density Shaking Cultures. Protein Expression Purif. 2005, 41, 207234.

(37) Dubourg, H.; Stines-Chaumeil, C.; Didierjean, C.; Talfournier, F.; Rahuel-Clermont, S.; Branlant, G.; Aubry, A. Expression,
Purification, Crystallization and Preliminary X-Ray Diffraction Data of Methylmalonate-Semialdehyde Dehydrogenase from Bacillus Subtilis. Acta Crystallogr., Sect. D: Biol. Crystallogr. 2004, 60, 14351437.

(38) Case, D. A.; Darden, T. E.; Cheatham, I. T. E.; Simmerling, C. L.; Wang, J.; Duke, R. E.; Luo, R.; Merz, K. M.; Pearlman, D. A.; Crowley, M.; Walker, R. C.; Zhang, W.; Wang, B.; Hayil, S.; Roitberg, A.; Seabra, G.; Wong, K. F.; Paesani, F.; Wu, X.; Brozell, S.; Tsui, V.; Gohlke, H.; Yang, L.; Tan, C.; Mongan, J.; Hornak, V.; Cui, G.; Beroza, P.; Mathews, D. H.; Schafmeister, C.; Ross, W. S.; Kollman, P. A. AMBER 9; University of California: San Francisco, CA, 2006.

(39) Duan, Y.; Wu, C.; Chowdhury, S.; Lee, M. C.; Xiong, G.; Zhang, W.; Yang, R.; Cieplak, P.; Luo, R.; Lee, T.; Caldwell, J.; Wang, J.; Kollman, P. A Point-Charge Force Field for Molecular Mechanics Simulations of Proteins Based on Condensed-Phase Quantum Mechanical Calculations. J. Comput. Chem. 2003, 24, 1999-2012.

(40) Wang, J.; Wolf, R. M.; Caldwell, J. W.; Kollman, P. A.; Case, D. A. Development and Testing of a General Amber Force Field. J. Comput. Chem. 2004, 25, 1157-1174.

(41) Bryce, R. Amber Parameter Database, 1998.

(42) Berendsen, H. J. C.; Postma, J. P. M.; van Gunsteren, W. F.; DiNola, A.; Haak, J. R. Molecular Dynamics with Coupling to an External Bath. J. Chem. Phys. 1984, 81, 3684-3690.

(43) Ryckaert, J.-P.; Ciccotti, G.; Berendsen, H. J. C. Numerical Integration of the Cartesian Equations of Motion of a System with Constraints: Molecular Dynamics of n-Alkanes. J. Comput. Phys. 1977, 23, 327-341.

(44) DeLano, W. L. The PyMOL Molecular Graphics System; San Carlos, CA.

(45) Talfournier, F.; Stines-Chaumeil, C.; Branlant, G. Methylmalonate-Semialdehyde Dehydrogenase from Bacillus Subtilis: Substrate Specificity and Coenzyme A Binding. J. Biol. Chem. 2011, 286, 21971-21981.

(46) Tuck, L. R.; Altenbach, K.; Ang, T. F.; Crawshaw, A. D.; Campopiano, D. J.; Clarke, D. J.; Marles-Wright, J. Insight into Coenzyme A Cofactor Binding and the Mechanism of Acyl-Transfer in an Acylating Aldehyde Dehydrogenase from Clostridium Phytofermentans. Sci. Rep. 2016, 6, 22108.

(47) Pal, D.; Chakrabarti, P. On Residues in the Disallowed Region of the Ramachandran Map. Biopolymers 2002, 63, 195-206. 